\title{
SayWhen: An automated method for high-accuracy speech onset detection
}

\author{
Peter A. Jansen And Scott Watter \\ McMaster University, Hamilton, Ontario, Canada
}

\begin{abstract}
Many researchers across many experimental domains utilize the latency of spoken responses as a dependent measure. These measurements are typically made using a voice key, an electronic device that monitors the amplitude of a voice signal, and detects when a predetermined threshold is crossed. Unfortunately, voice keys have been repeatedly shown to be alarmingly errorful and biased in accurately detecting speech onset latencies. We present SayWhen - an easy-to-use software system for offline speech onset latency measurement that (1) automatically detects speech onset latencies with high accuracy, well beyond voice key performance, (2) automatically detects and flags a subset of trials most likely to have mismeasured onsets, for optional manual checking, and (3) implements a graphical user interface that greatly speeds and facilitates the checking and correction of this flagged subset of trials. This automatic-plus-selective-checking method approaches the gold standard performance of full manual coding in a small fraction of the time.
\end{abstract}

The latency of spoken responses is used as a dependent measure in many domains of experimental psychology. Measurement of vocal response onset in the contemporary literature is typically achieved with a voice key-an electronic device that in its most basic form monitors the amplitude of an acoustic (voice) signal on a given trial, and is triggered when this signal first exceeds a predetermined amplitude threshold. Voice keys are commonly used as reaction time (RT) measurement instruments in behavioral experiments examining a wide range of topics, including but certainly not limited to attention (e.g., De Houwer, 2004; Naccache et al., 2005), memory (e.g., Nino \& Rickard, 2003; Rohrer \& Pashler, 2003), and emotion (e.g., Carroll \& Young, 2005; Meier \& Robinson, 2004). In a recent survey of representative publications, $95 \%$ of articles studying naming latencies used a voice key to quantify them (Rastle \& Davis, 2002). The availability and ease of implementation of voice keys as response measurement devices is further simplified by modern experimental software such as Presentation, www.neurobs.com; E-Prime, www.pstnet.com, which implement voice key functionality via a standard computer audio interface.

Despite their widespread use, voice keys have a number of potentially serious shortcomings; put bluntly, they appear to be inconsistent and error prone in accurately measuring speech onset latencies. Such problems have been suspected for many years now (e.g., Pechmann, Reetz, \& Zerbst, 1989). Recently, Kessler, Treiman, and Mullennix (2002) provided a direct and extensive examination of some of the phonological issues associated with measuring onset latencies, and the subsequent effects on accuracy of voice key measurements. The initial phoneme of a spoken response was found to substantially and systematically bias voice key detection latency, with smaller but still consistent influences from second phonemes. Rastle and Davis (2002) describe the case of Kawamoto and Kello (1999), who found that words with complex onsets (e.g., spat, /sp/) are produced faster than those with simple onsets (e.g., sat, /s/), in opposition to Frederiksen and Kroll (1976), who reported the reverse. The former used an offline software measurement algorithm, the latter a voice key. Rastle and Davis repeated this experiment, measuring vocal response latencies with a typical amplitude-threshold voice key, and comparing these values to the gold standard of hand coding speech onsets via visual inspection of digitized waveform data. They found marked differences between these two measurement methods, with hand coding supporting Kawamoto and Kello and voice key data supporting Frederiksen and Kroll - a stark demonstration of the potential perils of relying on voice key data.

A number of measures have been proposed to deal with these problems. As the best alternative available, Kessler et al. (2002) advise matching the phonology of to-bespoken words across conditions on as much of the head (onset) of the stimuli as possible. Whereas this probably helps reduce variability in voice key measurement error, Rastle and Davis (2002) have demonstrated that severe problems can arise, despite a moderate amount of this kind of control. Another proposed solution is the integrator voice key. Instead of simply setting a fixed amplitude threshold for speech onset detection, integrator voice keys are essentially sensitive to the cumulative amount of signal present within a specified time window-this allows slowly increasing, low-amplitude signals (e.g., the onset

P. A. Jansen, jansenpa@mcmaster.ca or S.Watter, watter@mcmaster.ca 
$/ \mathrm{s} /$ in the word sat) to trigger a response prior to the highamplitude onset of the voiced vowel, due to the accumulation of a low-amplitude but consistent signal. Integrator voice keys may offer some improvement over traditional amplitude threshold voice keys, but they seem far from an ideal solution. In their comparison of simple versus complex onsets, Rastle and Davis also assessed integrator voice key performance. They found that although it yielded smaller measurement errors (as compared with hand coding) than did a traditional amplitude-threshold voice key, the resulting pattern of data (a null result) was still substantially different than that from hand-coded data.

A third and somewhat obvious solution is that of measuring and coding speech latency data offline by hand, using visual displays of the recorded waveforms. Although this seems the natural gold standard, few researchers take this approach, probably because of the very large costs in time and effort, as well as the technical issues involved in recording, visualizing and manipulating these data. We suggest that most of these technical difficulties are easily solved nowadays, with low-cost computer hardware allowing direct digital recording of responses, and high-quality free and open-source visualization tools allowing easy and simple inspection of waveforms (e.g., Audacity, audacity .sourceforge.net; Praat, www.praat.org). However, even with these tools, manual measurement is still exceptionally time consuming, and thus a dilemma arises: Do we continue to rely on voice key data, given accumulating evidence of pervasive error and bias, or do we spend many times the duration of an experiment's data collection hand coding every trial? We present here a third alternative - an easy-touse automated software algorithm for offline speech onset latency measurement that approaches the accuracy of hand coding while being vastly more time efficient.

Our SayWhen software package, described in detail below, combines a sensitive speech onset detection algorithm with a number of additional heuristics that (1) enable the program to automatically identify speech onsets for a large proportion of trials with accuracy approaching that of hand coding, and (2) allow the program to automatically flag a subset of trials whose onset latencies are more likely to have been mismeasured, for optional subsequent human verification. This automatic measurement system is paired with an interactive graphical user interface, designed to speed and facilitate the manual inspection and adjustment of speech onset measurements via visualization of the recorded acoustic waveforms of individual trials. Our system allows the operator a choice between fully automatic operation (simply use the data automatically generated), fully manual operation (inspect every trial, facilitated by our interface), or a hybrid state, where the program itself flags and presents to the user only the subset of trials with speech onsets that it suspects it has done a poor job of measuring. It is in this hybrid state that our program is most useful, and allows the user an optimum balance between coding time and accuracy. In its worst case, with no user intervention or correction, the algorithm automatically tags speech onsets far more accurately than a voice key, generally taking only a few minutes of computation per experimental session. At its best, our program combines partial automaticity while tagging problem trials for human attention, resulting in near-handcoded accuracy in a fraction of the time. We describe our system in detail below, then compare its performance on a large experimental data set from our laboratory with both amplitude threshold voice key and hand-coded data.

\section{PROGRAM ALGORITHM AND DESCRIPTION}

\section{Goals}

In light of the known limitations in measurement accuracy and bias of modern voice keys, as well as the substantial effort required to hand code speech onset latency for subject data, we set out to develop a largely automated method of speech onset detection and latency measurement that would combine substantial accuracy improvements over existing automated methods (principally voice key solutions), while maintaining their speed of implementation. A number of other technical solutions to this general problem have been proposed, including other software solutions (e.g., Runword; Kello \& Kawamoto, 1998), and a recent hardware solution improving on simple amplitude threshold detection (Tyler, Tyler, \& Burnham, 2005). In developing our current software solution, we had two main goals: to develop an accurate automatic speech onset detection algorithm; and to develop a graphical user interface for this software that would facilitate and optimize the task of manually inspecting and checking speech onset latencies in individual trial waveforms, and that was both easy to use and adaptable to a wide range of experimental situations and methodologies.

\section{Algorithms}

The SayWhen speech onset detection algorithm is essentially the combination of a highly sensitive signal detection facility with a number of simple heuristics for discerning whether a detected signal is likely to be noise, or the onset of a speech signal. Together, these elements provide an elegant method for signal detection, while incorporating a few known considerations for noise when recording both from human subjects and in a nonideal physical environment. Within a given trial, the overall onset detection algorithm proceeds in order, as follows: (1) candidate signal detection; (2) short-duration noise detection; (3) discontinuous phonation detection; (4) frequency look-back-through-time; and (5) problem trial tagging. Each of these mechanisms will now be described in detail.

Candidate signal detection. The algorithm's initial candidate signal detection is somewhat analogous to traditional voice key detection, which makes use of an amplitude threshold device. Typically, an amplitude threshold based detection scheme functions by progressively scanning a signal in time for signals whose amplitude surpasses some preset value, for some nonzero amount of time. An integration-based voice key extends this method across time, where the sum of amplitudes across some small period of time must exceed the amplitude threshold before a signal is said to be detected. The candidate signal 
detection algorithm makes use of a conceptually similar method of thresholding, but examines the signal in the frequency domain rather than in amplitude.

The algorithm makes use of the fast Fourier transform (FFT), an efficient implementation of Fourier analysis, to transform a discrete window of size $n$ samples in the audio recording into the frequency domain; or, more specifically, a set of sinusoidal waves with known periods and amplitudes that sum to the input signal. Operating under the assumption that the majority of the experimental recording will be quiet (with the exception of speech signals and noise), the window begins from trial onset and is initially set to be relatively large. The Fourier spectrum is searched for signals in any given frequency component above a certain threshold. If no signals are found above the threshold, the window location increments ahead slightly in time and searches again. This process continues until either a signal is located above a certain threshold, or until a set limit on the search distance is reached.

When a signal peak is identified, the window momentarily stops scanning ahead in time and decreases the size of the scanning window to half of its current value. Scanning ahead in time then resumes until a signal above the threshold is again detected and the scanning window size is again decreased by half. This process continues until the scanning window becomes very small. For instance, on a $44.1-\mathrm{kHz}$.wav recording, the scanning window may initially be set to 1,024 samples (nearly $1 / 44 \mathrm{sec}$, or $23 \mathrm{msec}$ ), with a lower bound of 16 samples (nearly $1 / 3 \mathrm{msec}$ ). In this way, even low-amplitude signals with one or more strong fundamental frequencies can be detected extremely accurately. Once the lower bound of the Fourier transform window size has been reached, and a signal above the threshold is detected, the algorithm makes note of the time index of this candidate signal and passes it further on in the overall algorithmic process. These later processes take the form of heuristics, which determine if the algorithm has located a signal with a high probability of being a speech onset, or whether the algorithm has likely encountered noise and should continue its search.

Short-duration noise detection. The first heuristic applied to the candidate speech signal was designed to check for short-duration noise in the audio recording. Even when precautions are taken and quality recording equipment is used in a low-noise environment, occasional noise, in the form of short-duration, low-amplitude spikes, occurs frequently over the course of an experimental session. Where these peaks are normally inaudible and below the detection threshold of voice keys, they often show up as tall peaks in the Fourier spectra, causing the algorithm to identify these peaks as candidate onset signals. The shortduration noise detection heuristic aims to reject these candidate signals by examining their amplitude profile.

A vast majority of phonemic onsets in speech signals quickly increase amplitude in a short period of time, and sustain this amplitude increase for temporally large regions of the spoken response. Using what is essentially an integration voice key in reverse, this heuristic sums the total change in amplitude from the candidate detection out to some short distance. This sum is then compared with a set noise threshold. Where the average signal amplitude in this region falls below this noise threshold, the candidate signal is classified as noise, and the onset detection process moves slightly ahead in time and continues searching for candidate signals. Where the candidate signal is not classified as noise, its status is upgraded to a detected signal, and processing proceeds to the next step in the overall algorithm.

Discontinuous phonation detection. Once a candidate signal onset has been positively identified, the program begins to focus on correcting any potential errors it may have made. In particular, this heuristic is interested in identifying cases where early low-amplitude phonemes were incorrectly categorized as noise and passed over, resulting in an erroneously larger onset latency. This occurs quite frequently for phonemes with low-amplitude gradual onsets, such as /s/ and /f/, as well as phonemes with a short initial burst followed by a brief period of silence, as is occasionally the case for velar onsets, such as $/ \mathrm{g} /$. In these cases, the algorithm very likely detected their signals earlier on but incorrectly classified them as noise.

To remedy the situation, the discontinuous phonation detection heuristic makes note of all cases where the algorithm detects candidate signals that are rejected by the short-duration noise heuristic. Then, when a signal first passes the noise detection heuristic, the algorithm checks this list of previously rejected signals. If a previously rejected signal is found within some short distance back from the candidate onset, the algorithm then considers the earliest detected onset within this short earlier distance as the new detected onset.

Frequency look-back-through-time. As a final computational step, the algorithm evaluates the Fourier spectrum of the region immediately following the detected onset out to some small distance. Any frequency peaks in the Fourier spectrum above a minimum threshold are noted before the algorithm begins to slowly move earlier in the recording, monitoring the activity of those peaks. When all but a few peaks drop below the detection threshold, the algorithm classifies the signal as having faded, and uses its final location as the detected signal onset. As most onsets have been detected very accurately by this stage, the total contribution of this heuristic is often quite low. However, this step is included both for its occasional significant contribution as well as for its completeness.

Problem trial tagging. Ultimately, if a detection algorithm isn't perfect, its usefulness is vastly increased if it is able to detect instances where it may not have performed very well. In a process similar to detecting short-duration noise, after a speech signal onset has been detected via the steps above, the algorithm checks the average amplitude from the detected onset out to some short distance forward in time. If this average amplitude is below some threshold, typically twice the value of the short-duration noise threshold, the signal is flagged as having a low-amplitude onset, possibly requiring human attention. Onsets that have extremely short or long RTs are also flagged for human attention.

\section{Implementation and Interface}

The SayWhen speech onset detection algorithm was originally developed in MATLAB to facilitate algorithm 
development and analysis before being ported to Visual $\mathrm{C}++$, a platform which facilitates program execution speed, ease of distribution, and the development of a graphical user interface. Whereas the original MATLAB implementation was completely automated, the addition of a graphical user interface allows users to view and verify detected onsets, quickly jump to onsets that have been flagged by the algorithm, and finally correct any discrepancies with speed and ease.

Figure 1 presents a schematic depiction of a basic behavioral experimental workflow using SayWhen for speech latency measurement. Upon loading a .wav audio recording of an experimental session, the onset detection algorithm automatically scans through the recording searching for speech onsets. Pragmatically, the detection of accurate RTs must be relative to some trial onset, which must be marked or otherwise recorded in the experimental data. In the present implementation, we have used a method whereby a trial onset marker generated by the stimulus presentation computer $(10 \mathrm{msec}, 1-\mathrm{kHz}$ square wave) is sent to one channel of a stereo audio recording at the onset of each trial. The SayWhen program is able to scan audio recordings for this trial onset signal, and to detect its onset very accurately. Once the file has been completely scanned for trial onsets, the file is scanned for speech onsets starting from the beginning of each detected trial onset. Once a speech onset is detected for a given trial, the program moves on to the next trial until no further trials remain, and the entire audio recording has been tagged.

Once this automated detection process has been completed, the user is free to view and manipulate the onset data. By default, the SayWhen software interface displays waveform data at a relatively high magnification, centered on the detected onset of the current trial, which is indicated with an overlaid position marker, akin to a standard wave editor. Figure 2 shows an example of the SayWhen user interface with a speech onset detected and marked, ready for user inspection and potential manual correction. The program provides information on current and total trial numbers, with summary statistics on trial counts, trials flagged for checking, and means and standard deviations $(S D \mathrm{~s})$ of automatically measured latencies, given several different flagging and trial exclusion criteria. The interface has dedicated intuitive controls to allow inspection of every trial onset, or to take the user only to the critical onset segment of subsequent trials flagged for human attention. If manual inspection of a trial reveals mismeasurement by the automated algorithm, the user can move or place the onset marker cursor directly at the correct onset point using the mouse. Auditory playback of the waveform from the current onset marker, or directly from any clickable point on the displayed waveform, is always available directly from the display interface, allowing auditory playback of different segments without necessarily changing marked onset positions. Speech onset latency data are reported via a text $\log$ file for each subject waveform, including the trial number and the automatic latency measurement, with hand-corrected measurements for particular trials (if any) added to the appropriate trials following manual inspection.

\section{PROGRAM PERFORMANCE}

The SayWhen speech onset detection software was tested with a large experimental data set acquired in a recent language production study in our laboratory, where subjects were asked to make speeded spoken free word

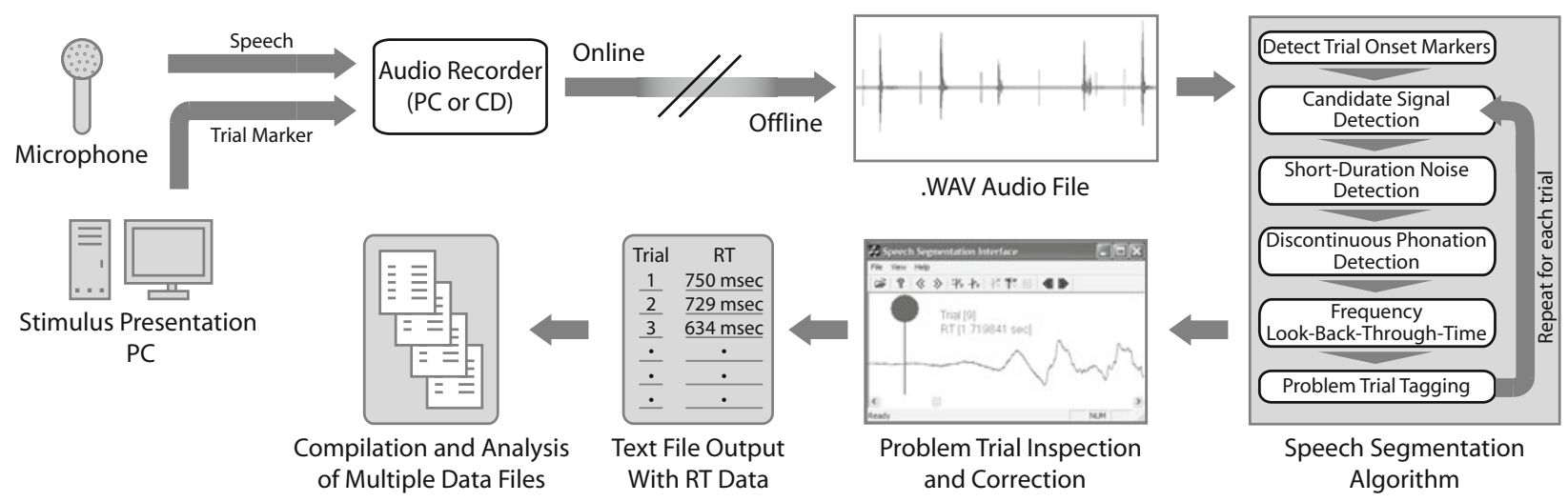

Figure 1. Schematic of voice latency measurement process using SayWhen software. Subjects' vocal responses to presented experimental stimuli are recorded along with a brief auditory signal generated by the stimulus computer at the start of each trial (10 msec, 1-kHz square wave; not audible to subjects), in order to time-lock subjects' responses to trial stimulus events. The SayWhen software calculates RT for each trial as the time difference between the onset of auditory markers and subjects' detected vocal onsets. Although our laboratory uses a separate digital audio recorder to record subject speech and stimulus computer timing markers, it is possible for the stimulus presentation computer to record these audio data with many modern experimental software tools. The digital audio file from this experimental session is later processed offline, using our SayWhen software. SayWhen initially detects all onset markers to identify trial boundaries, and then runs an iterative algorithm (described at length in this article) to identify speech onsets and measure vocal RT within each trial. Following this initial automatic detection process, the user can optionally inspect and correct the detected speech onsets via an interactive interface, including having the program guide the user through inspection of an automatically selected subset of trials flagged as most likely to have been mismeasured by the program. Automatic plus manually corrected RT data for each trial are available in a standard tabulated text file, ready for use with other data analysis tools. 


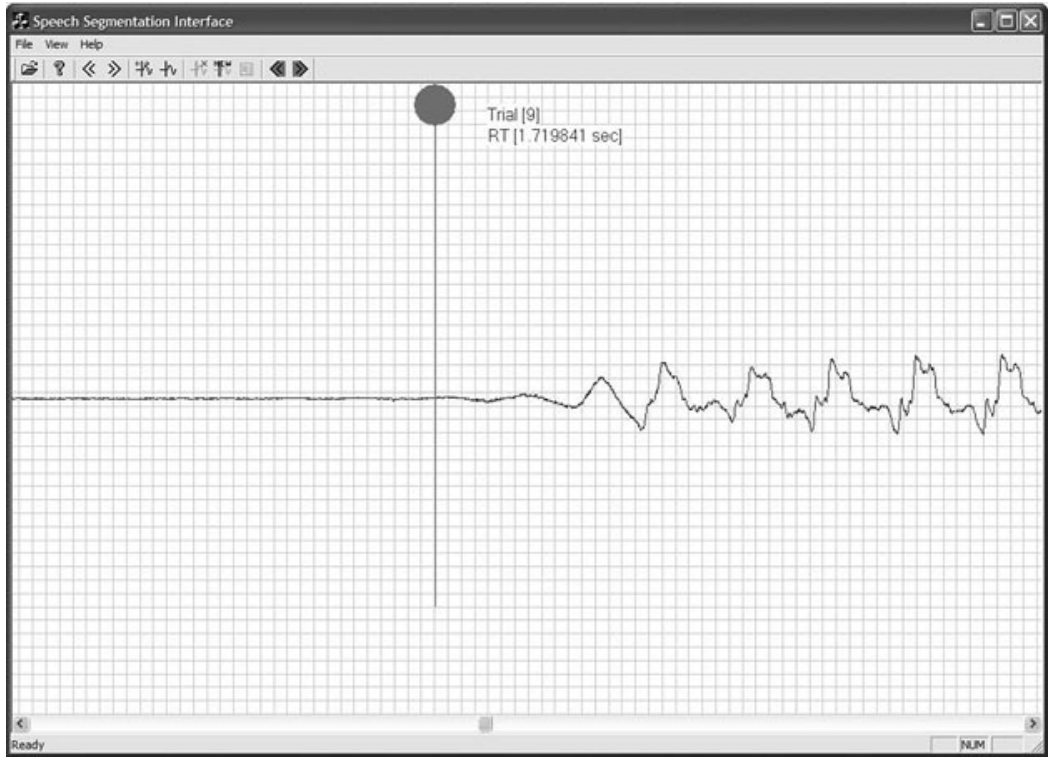

Figure 2. The SayWhen user interface. This example shows a sample waveform with the position cursor at the detected speech onset for a given trial. The user can reposition the onset marker for the trial directly with the mouse, and auditory playback of the current trial waveform from any specific starting point is available by clicking on the waveform at that point. Available toolbar controls (left to right) allow the user to open a .wav file, get program information, jump to the previous or next trial, edit the trial onset marker or jump to the detected speech onset of the current trial, delete or add a new trial onset marker, modify detection algorithm parameters, and jump to the previous or next flagged trial. This interface allows the user to rapidly inspect the automatically detected vocal onsets for each trial if desired, but also allows for the guided inspection of an automatically flagged subset of trials judged by the program as the most likely to have been mismeasured by the SayWhen voice onset detection algorithm.

association responses to presented word stimuli. This data set had the virtue of both having been automatically tagged by a typical amplitude threshold voice key under experimental conditions during subject testing, and having been later hand coded from recorded digital audio files by trained undergraduate research assistants. Of the 28 subjects initially studied, 4 were excluded from our analysis due to equipment failure (trial onset markers not recorded) or high levels of background noise. In total, 3,940 trials across 24 subjects were analyzed. Details of our algorithm parameters are given in the Appendix.

In order to determine the benefit of our speech onset detection algorithm over a voice key, we compared the voice key data and data from our SayWhen algorithm against hand-coded data in three separate tests: (1) fully automatic, where the output of our speech onset detection algorithm was used without any checking or modification; (2) automatic, excluding very short or long RTs, where trials with speech onset detections below or above predetermined latency thresholds were excluded; and (3) automatic, excluding all flagged trials, which gives an estimate of the best possible performance of our program when used in its hybrid mode - that is, assuming that trials that have been flagged as potentially requiring human attention are manually inspected and corrected to within hand-coding accuracy as necessary.
The results are shown in Figure 3. This histogram reflects the relative proportions and distribution of difference scores between human hand coding, taken in this case as the gold standard, and either voice key or algorithm performance in the various conditions. In this case, a lower score reflects closer agreement between human coding and the automated methods. Histograms were initially constructed from individual subjects' data, and then averaged across subjects within each bin, in order to show some measure of how well a voice key and our algorithm performed over data from a range of different subjects. As expected, voice key performance was generally poor, with only $32.9 \%$ of trials being tagged within $5 \mathrm{msec}$ of hand coding, and $41.7 \%$ within $10 \mathrm{msec}$. Additionally, $44.8 \%$ of trials disagreed with hand coding by $25 \mathrm{msec}$ or greater-in essence, the voice key is correctly detecting onsets less often than it is tagging them with substantially incorrect values.

The SayWhen software algorithm performed considerably better than a voice key in all cases. In the fully automatic case (no user checking or intervention), across all trials, $59.6 \%$ of trials were tagged within $5 \mathrm{msec}$ of hand coding, with $69.5 \%$ tagged within $10 \mathrm{msec}$. In this case, $18.6 \%$ of trials disagreed with hand coding by $25 \mathrm{msec}$ or greater - a factor of 2.4 improvement over the voice key. When trials flagged as having unusually short or long RTs were omitted (not shown in Figure 3), algorithm per- 


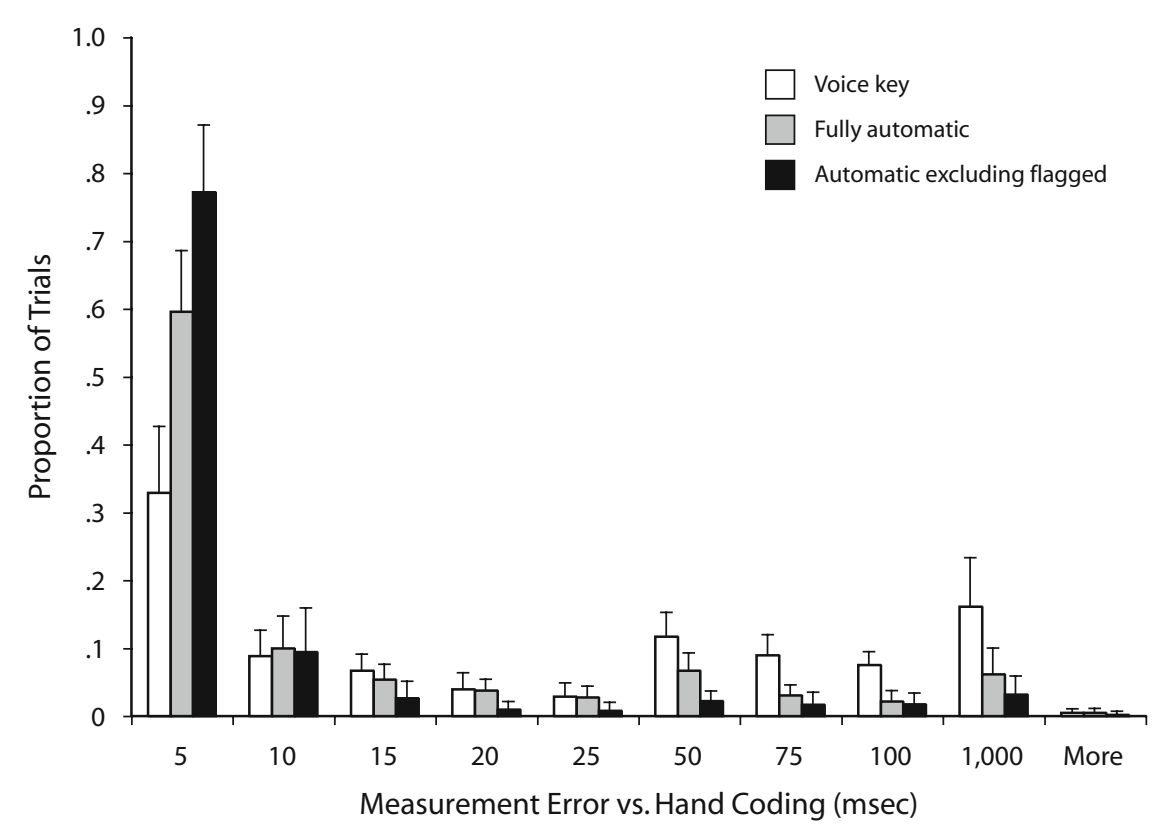

Figure 3. Histogram of proportions of speech onset measurement errors arising from a standard amplitude-threshold voice key (white), a fully automatic application of our onset detection algorithm (gray), and our automatic algorithm excluding the trials that the algorithm itself flagged as being potentially error-prone (black). Measurement error is represented on the $\boldsymbol{x}$-axis, calculated as the difference in milliseconds between the respective measurement methods above and the gold-standard method of hand coding using visual inspection of digitized waveform data. Histogram bins represent trials with absolute error values equal to or less than the label value, but greater than the next smallest bin label. Proportions add to 1.0 for each of the three measurement methods. Error bars represent one standard deviation. The SayWhen automated onset detection algorithm performs substantially better than a standard voice key, with additionally superior accuracy available via guided manual checking of automatically flagged trials.

formance remained essentially unchanged, with $60.6 \%$ of trials falling within $5 \mathrm{msec}$ of hand coding, and $70.7 \%$ falling within $10 \mathrm{msec}$. An additional $16.9 \%$ of trials differ with hand coding by $25 \mathrm{msec}$ or greater. Given that a total of $213(5.4 \%)$ trials were excluded by these criteria, this small change is not surprising.

The exclusion of all flagged trials (detected as slowonset, mainly sibilant or fricative initial phonemes) saw a drastic increase in performance, with $77.2 \%$ of trials tagged within $5 \mathrm{msec}$ of hand coding, and $86.6 \%$ falling within $10 \mathrm{msec}$. In this case, $9 \%$ of trials disagreed with hand coding by $25 \mathrm{msec}$ or greater - a factor of 5 improvement over a voice key. Furthermore, this performance includes only those trials not flagged for human attention and checking - if one assumes using our hybrid method of automatic measurement plus facilitated selective manual checking, the remainder of trials will likely be coded with high accuracy, elevating this performance even further. This gives an expected accuracy of almost $90 \%$ of trials coded to within $5 \mathrm{msec}$ of hand coding performance, with another approximately $4.5 \%$ within $10 \mathrm{msec}$, but at the obvious additional cost of manually checking a large number of additional trials.

The automatic detection performance of our SayWhen algorithm is comparable to the very good onset detection performance of Kello and Kawamoto's (1998) Runword software. Although our data are not completely alignable, we can make several approximate comparisons that demonstrate similar levels of performance. Kello and Kawamoto reported detection accuracy for stimuli with sibilant onsets as a mean difference of $-4.2 \mathrm{msec}$ versus handcoded data, with an $S D$ of $10.1 \mathrm{msec}$. Assuming a roughly Gaussian distribution of individual responses, approximately $68 \%$ of responses should fall between $-14.3 \mathrm{msec}$ and $5.9 \mathrm{msec}$ - our data (grouped as absolute error) show overall performance with less than $10 \mathrm{msec}$ of error at $69.5 \%$. Excluding trials with low-amplitude onsets, our algorithm's performance was again comparable to Kello and Kawamoto's bisyllabic naming condition (only one low-amplitude onset stimulus included), with a mean difference from hand coding of $0.5 \mathrm{msec}$ with an $S D$ of $4.7 \mathrm{msec}$, suggesting approximately $68 \%$ of trials within -4.2 to $5.2 \mathrm{msec}$ - again comparable to our algorithm's performance of $77.2 \%$ of trials falling within $5 \mathrm{msec}$ of hand coding.

Unfortunately for our experiment, 1,838 trials - nearly half of all data - were flagged for human attention. Although this may seem to be only a minor improvement over completely manual coding, several factors substantially mitigate this process here. First, we provide an integrated graphical interface for viewing trial waveforms with automatically detected onset positions, and the facility to 
either edit that onset with a graphical cursor onscreen or to jump to the next flagged trial - a system that drastically speeds up manual checking and editing. Second, our detection parameters serve to maximize sensitivity to trials that might be mismeasured. In its present incarnation, our program flags all trials with low-amplitude onsets for subsequent human attention, even though it does a very accurate job on a majority of these trials. Of all trials flagged by this heuristic, $49.6 \%$ were within $5 \mathrm{msec}$ of hand coding, and $60.9 \%$ were within $10 \mathrm{msec}$. In practical terms, our program allows the rapid inspection and verification of speech onset data, taking only a few seconds to visually verify or graphically correct each flagged trial. As an example of its facilitation of this kind of activity: In performing these analyses, we used our program to selectively find and inspect flagged trials from every subject, as part of assessing what kinds of trial events lead to poor speech onset detection by our algorithm. A single user inexperienced in using our interface was able to inspect and correct as necessary an entire subject's experimental session (168 trials: approximately $12 \mathrm{~min}$ ) in approximately 5 min with high accuracy, as reported above.

Overall, a total of 190 trials (5.1\%) differed by $25 \mathrm{msec}$ or greater between automatic and hand coding and were not flagged for human attention by our algorithm. This represents the catastrophic failure rate of our automated method, even when all flagged trials are reviewed. These trials compose the right hand tail of the best-performance (nonflagged) histogram in Figure 3. Of these failed-detection trials, approximately one third represent cases where the algorithm failed to detect extremely low onset phonemes, where the initial amplitude of the phoneme was on the order of the signal-to-noise ratio of the recording. The remaining two thirds of errors largely consist of nonspeech noise detected as a speech onset, arising from subject-produced noise including lip-smacks and microphone/headset movement, or other unknown environmental noise. Measurement errors greater than $100 \mathrm{msec}$ were almost exclusively due to this latter mechanism.

\section{DISCUSSION}

We present SayWhen, a software program to automatically measure speech onset latencies from recorded audio data, that combines high accuracy with speed and ease of use. Our program can be used in a number of ways, depending on the degree of measurement accuracy desired. In its fully automatic mode, SayWhen uses a sensitive speech onset detection algorithm, coupled with a number of heuristics, to measure speech onset latencies with comparable accuracy to Kello and Kawamoto's (1998) Runword program. This performance is substantially better than a traditional amplitude threshold voice key.

In addition to this automatic onset latency measurement of all trials, SayWhen identifies a subset of trials most likely to have the greatest measurement error and provides a streamlined graphical interface to facilitate the rapid review and correction as necessary of mismeasured onsets. Using our program in this hybrid way affords an additional substantial improvement in measurement accuracy over and above fully automatic onset detection, and approaches the gold standard performance of full hand coding of all data. Using this hybrid automatic plus selective checking method, a majority of trials with errors in onset detection measurement greater than $10 \mathrm{msec}$ and up to approximately $75 \mathrm{msec}$ appear to be identified and flagged for human attention, represented in Figure 3 as the difference between automatic (gray) and automatic minus flagged (black) trial proportions in 15- to 50-msec bins, representing approximately $15 \%-20 \%$ of all trials in this study.

Under this best hybrid performance, approximately $5 \%$ of all trials were found to have greater than $25-\mathrm{msec}$ onset measurement error compared with hand coding, while not being flagged for human attention, representing a complete failure of our algorithm on these trials. Upon inspection, two thirds of these trials contained substantial nonspeech sounds prior to vocal response onset, erroneously detected as speech onsets. This kind of measurement error is similarly likely to occur with voice keys and other software systems. Short of a substantially more complex algorithm to distinguish speech versus nonspeech sounds on the basis of inherent frequency components (for example), or an experimenter noticing the occurrence of extraneous noise during data collection for these trials, offline manual human inspection is the only way to reliably detect these trials.

Although our present solution provides a substantial improvement to voice onset latency measurement, its performance is not perfect. Changes or additions to the algorithms embodied in our SayWhen software could likely improve its performance even further. As one example, standard voice keys are in a sense unable to "see the forest for the trees"; that is, they progressively scan temporally fine regions of an audio signal, with little or no information as to whether a currently detected above-threshold signal is part of a much greater whole or merely transient noise. Our present algorithm departs from this approach, using different heuristics to scan the Fourier-transformed auditory signal both forwards and backwards in time, giving more accurate and more selective onset detection performance. Extending this approach, one might imagine that a next-generation algorithm could first scan an entire trial, and identify the first continuous, high-amplitude region of signal (most likely speech). The algorithm could then use heuristics similar to those outlined here that would scan progressively earlier in time to identify a precise onset time, while compensating for low-amplitude phonemes and discontinuous phonation. Such a method has the potential to be immune to most early trial noise, including nonspeech noise from both subjects and the environment, where the duration of this noise is less than a few hundred milliseconds. Considering that this kind of nonspeech noise was the primary source of catastrophic failure for our present algorithm, this approach would likely yield an improvement in overall program performance, and could substantially improve SayWhen's resistance to identifying early nonspeech noises as vocal onsets.

In summary, we have developed a software program that provides a very accurate and fully automatic method for measuring speech onset latencies from recorded audio 
data. It is both easy to use and easy to implement in a wide range of experimental settings. All that is required is an audio recording, of reasonable quality, of subjects' responses, with auditory trial onset markers included. If necessary, our program can be modified to analyze trial data stored as separate individual audio files, or to use different types of trial onset markers. For studies that just use a voice key without hand coding-likely to be the majority of cases - or other situations that may not afford a great deal of time for analysis, measurement accuracy could be vastly improved simply by using our SayWhen speech onset detection algorithm in fully automatic mode. For studies in which researchers would otherwise hand code subjects' speech onset data, SayWhen offers a way to achieve very-close-to-hand-coded accuracy in a small fraction of the time typically required.

\section{AUTHOR NOTE}

We thank C. Boyd, K. R. Humphreys, and the members of the Cognitive Science Laboratory at the Department of Psychology, Neuroscience and Behaviour, McMaster University, for their assistance in the development of this work. This project was supported by Natural Science and Engineering Research Council of Canada (NSERC) Grant 327454 to S.W. Our speech onset detection software is available from our Web site, cogsci.mcmaster.ca, or by contacting the authors. Correspondence concerning this article should be addressed to P. A. Jansen or S. Watter, Department of Psychology, Neuroscience and Behaviour, McMaster University, 1280 Main Street West, Hamilton, ON, L8S 4K1 Canada (e-mail: jansenpa@mcmaster.ca orwatter@mcmaster.ca).

\section{REFERENCES}

Carroll, N. C., \& Young, A. W. (2005). Priming of emotion recognition. Quarterly Journal of Experimental Psychology, 58A, 1173-1197.
De Houwer, J. (2004). Spatial Simon effects with nonspatial responses. Psychonomic Bulletin \& Review, 11, 49-53.

Frederiksen, J. R., \& Kroll, J. F. (1976). Spelling and sound: Approaches to the internal lexicon. Journal of Experimental Psychology: Human Perception \& Performance, 2, 361-379.

Kawamoto, A. H., \& Kello, C. T. (1999). Effect of onset cluster complexity in speeded naming: A test of rule-based approaches. Journal of Experimental Psychology: Human Perception \& Performance, 25, 361-375.

KeLlo, C. T., \& Kawamoto, A. H. (1998). Runword: An IBM-PC software package for the collection and acoustic analysis of speeded naming responses. Behavior Research Methods, Instruments, \& Computers, 30, 371-383.

Kessler, B., Treiman, R., \& Mullennix, J. (2002). Phonetic biases in voice key response time measurements. Journal of Memory \& Language, 47, 145-171.

Meier, B. P., \& Robinson, M. D. (2004). Why the sunny side is up: Associations between affect and vertical position. Psychological Science, 15, 243-247.

Naccache, L., Dehaene, S., Cohen, L., Habert, M.-O., GuichartGomez, E., Galanaud, D., \& Willer, J.-C. (2005). Effortless control: Executive attention and conscious feeling of mental effort are dissociable. Neuropsychologia, 43, 1318-1328.

Nino, R. S., \& RickARD, T. C. (2003). Practice effects on two memory retrievals from a single cue. Journal of Experimental Psychology: Learning, Memory, \& Cognition, 29, 373-388.

Pechmann, T., Reetz, H., \&Zerbst, D. (1989). Kritik einer Meßmethode: Zur Ungenauigkeit von voice-key Messungen [Critique of a method of measurement: On the unreliability of voice-key measurements]. Sprache \& Kognition, 8, 65-71.

Rastle, K., \& Davis, M. H. (2002). On the complexities of measuring naming. Journal of Experimental Psychology: Human Perception \& Performance, 28, 307-314.

Rohrer, D., \& PASHLER, H. E. (2003). Concurrent task effects on memory retrieval. Psychonomic Bulletin \& Review, 10, 96-103.

Tyler, M. D., Tyler, L., \& Burnham, D. K. (2005). The delayed trigger voice key: An improved analogue voice key for psycholinguistic research. Behavior Research Methods, 37, 139-147.

\section{APPENDIX}

In each case, the SayWhen speech onset detection software tagged all experimental data using the following algorithm-specific parameters. Initial onset detection: initial window size, 512 samples per channel; minimum window size, 16 samples per channel; FFT signal threshold, 8,000. Short duration noise threshold: 180 average over 750 samples per channel; silence period: 250 samples per channel. Discontinuous phoneme detection: 1,250 sample lookback. Frequency look-back-through-time: window size, 32 samples per channel; FFT signal threshold, 100. Lowamplitude (flagged) phoneme detection: window size, 500 samples per channel; threshold, 400. Reaction time cutoffs: minimum, $600 \mathrm{msec}$, maximum, 2,500 msec. All experiment recordings were digitized as $44.1 \mathrm{kHz} 16-$ bit .wav recordings. 